\title{
La política monetaria en presencia de las finanzas islámicas: un estudio exploratorio
}

\author{
Joudar Fadoua, Doctora investigadora \\ Dinar Brahim, Doctor y Profesor \\ Facultad de Economía y Gestión, \\ Universidad Hassan First, Settat, Marruecos
}

Doi:10.19044/esj.2021.v17n23p307

Submitted: 01 May 2021

Accepted: 16 July 2021

Published: 31 July 2021
Copyright 2021 Author(s)

Under Creative Commons BY-NC-ND

4.0 OPEN ACCESS

Cite As:

Fadoua J. \& Brahim D. (2021). La política monetaria en presencia de las finanzas islámicas: un estudio exploratorio. European Scientific Journal, ESJ, 17(23), 307.

https://doi.org/10.19044/esj.2021.v17n23p307

\section{Resumen}

En los últimos años se ha producido un verdadero desarrollo de la industria financiera islámica, abriendo nuevas oportunidades de financiación para los inversores. Para beneficiarse de sus productos, varios países se han embarcado en el experimento financiero islámico. Al contar ya con una infraestructura bancaria y monetaria convencional, la implantación de instituciones islámicas puede resultar difícil, ya que se supone que deben cumplir con la sharia al tiempo que operan junto a sus homólogos convencionales. Sin embargo, cuando los dos sistemas financieros coexisten, la práctica de la política monetaria es un verdadero desafío que da lugar a muchas reflexiones. De hecho, la prohibición de ciertas prácticas, como el uso de tipos de interés, por parte de las finanzas islámicas crea una necesidad real de diseñar instrumentos que se adecuen a los preceptos de la Sharia.

Palabras-clave : Política monetaria- Instrumentos- Finanzas islámicasFinanzas convencionales 


\title{
Monetary policy in the presence of Islamic finance: An exploratory study
}

\author{
Joudar Fadoua, Doctora investigadora \\ Dinar Brahim, Doctor y Profesor \\ Facultad de Economía y Gestión, \\ Universidad Hassan First, Settat, Marruecos
}

\begin{abstract}
In recent years, the Islamic financial industry has developed significantly, opening up new financing opportunities for investors. To benefit from its products, several countries have embarked on the Islamic financial experiment. Already having a conventional banking and monetary infrastructure, the implementation of Islamic institutions can be difficult, as they are supposed to be Sharia-compliant while operating side by side with their conventional counterparts. However, when the two financial systems coexist, the practice of monetary policy is a challenging and thoughtprovoking one. Indeed, the prohibition of certain practices, such as the use of interest rates, by Islamic finance creates a real need to design instruments that are appropriate to Shariah precepts.
\end{abstract}

Keywords: Monetary Policy, Instruments, Islamic Finance, Conventional Finance

\section{Introduction}

La crisis financiera puso en entredicho los principios de las finanzas convencionales, una crisis que comenzó a mediados de 2007 y que provocó una considerable inestabilidad económica en Estados Unidos y posteriormente en el resto del mundo. Fuertemente, la crisis de las hipotecas de alto riesgo se debe principalmente a la subestimación de los riesgos, la alta especulación, la falta de una regulación adecuada, así como las demás transacciones financieras que siguieron, como la titulización y la emisión de obligaciones con garantías de préstamos para obtener enormes beneficios a través de los derivados financieros.

La principal observación establecida desde la aparición de esta crisis es que sus principales causas radican en las tensiones del sistema financiero internacional, cuestionadas durante siglos; el sistema de intereses está ciertamente en el origen de las crisis bancarias, monetarias, financieras y económicas que conducen a la inestabilidad a todos los niveles, en particular 
a la inestabilidad financiera, que justifica el sobreendeudamiento de los hogares y las fuertes deudas públicas.

Así, el actual entorno financiero global fomenta cada vez más la discusión y el debate sobre métodos alternativos de financiación islámica. En un momento en que la crisis financiera ha dejado al descubierto los límites del sistema financiero convencional, su homólogo islámico se está imponiendo en el entorno de las decisiones financieras.

Las diferencias entre el modelo convencional y el modelo islámico representan diferencias fundamentales.

De hecho, la prohibición de intereses constituye la principal diferencia entre las finanzas convencionales y las finanzas islámicas. De esta manera, los países que han optado por un sistema conforme a la Sharia, han rediseñado sus sistemas bancarios para que sean adaptados a las transacciones financieras sin intereses. Estas medidas se han aplicado en países que han optado por un sistema dual en el que los bancos islámicos coexisten con los bancos convencionales, y en países que han islamizado sus sistemas bancarios como Irán y Pakistán. (Khan \& Mirakhor, 1989).

Además, y debido a las peculiaridades del sistema convencional que no son las mismas que las del sistema islámico. La valoración, las funciones y el papel del dinero son diferentes en este modelo financiero.

En consecuencia, existe una gran diferencia entre la percepción del dinero por parte del sistema financiero islámico y por el modelo convencional. En el sistema convencional, el dinero se asimila a un bien que se puede vender, comprar/alquilar y especular libremente. Tiene un valor temporal que se concreta en los intereses.

Sin embargo, cuando se trata del sistema financiero islámico, este punto de vista no es aceptado debido a sus fundamentos y cómo funciona. Así, la utilidad y la finalidad del dinero requieren un aspecto especial. Según los expertos en finanzas islámicas, el dinero es una unidad de cuenta y un medio de intercambio, porque el dinero como tal no debería cumplir ninguna función, razón por la cual el acaparamiento está prohibido.( Ayub, 2007 ; Ahmad \& Hassan, 2006).

Dicho esto, la política monetaria en presencia de bancos islámicos debería representar un verdadero desafío para los países con sistemas financieros duales. De hecho, el problema que se plantea es que los bancos centrales utilizan instrumentos monetarios muy peculiares, que pueden no estar en consonancia con los principios del modelo islámico. Lo que incita a 
reflexionar en otros sustitutos. Sin embargo, los bancos islámicos están presentes en dos tipos de sistemas financieros:

- Los sistemas financieros duales, en los que los bancos islámicos, coexisten con los bancos convencionales, como los países del Consejo de Cooperación del Golfo (CCG)

- Y sistemas financieros totalmente islamizados como Pakistán, Sudán e Irán.

En consecuencia, a través de este artículo, intentaremos responder a las preguntas siguientes :

- ¿Cuáles son los instrumentos de política monetaria que pueden sustituir a los instrumentos habituales en un sistema financiero totalmente islamizado ?

- ¿Y con qué instrumentos debería operar la política monetaria en un sistema financiero en el que coexisten los dos tipos de bancos?

Para ello, este artículo se presentará en dos partes principales. La primera parte estará dedicada a los instrumentos de la política monetaria en el sistema financiero islámico propuestos por la literatura, por un lado, y a los canales de transmisión de la política monetaria en presencia de los bancos islámicos, por otro lado. La segunda parte estará dedicada a un estudio exploratorio para identificar los diferentes instrumentos de la política monetaria en el caso de un sistema financiero dual (los países del CCG) y en el caso de un sistema financiero islamizado (Irán).

\section{La política monetaria y el sistema financiero islámico:}

La política monetaria tiene una gran importancia porque contribuye a mantener la estabilidad del sistema financiero. Por lo tanto, la pregunta que plantearemos a este nivel, es examinar cómo funciona la política monetaria en un sistema sin de intereses. Es evidente que los instrumentos basados en la tasa de interés no serían adecuados para los sistemas financieros islámicos, y habría que encontrar instrumentos que se ajustaran a los principios de la Shariah.

\subsection{Los instrumentos de la política monetaria en un sistema financiero islámico: revisión de la literatura}

Según Zangeneh y Salam (1993), el banco central puede utilizar muchas de las herramientas disponibles en un contexto bancario convencional. 
Sin embargo, algunos requieren modificaciones que pueden resultar costosas. Consideran que sería más eficaz mantener las mismas herramientas.

Sin embargo, algunos instrumentos, como la tasa de descuento que utilizan los bancos para controlar el volumen de crédito en una economía, son inaceptables en la banca islámica.

Esta es la perspectiva del estudio desarrollado por Khan y Mirakhor (1989), lo que ha tenido como objetivo desarrollar un modelo macroeconómico para ilustrar el funcionamiento de la política monetaria en un sistema financiero islámico. Este estudio concluye que a pesar de la adopción de instrumentos monetarios distintos a los aplicados por el sistema convencional, el impacto de la política monetaria sobre las variables económicas del sistema financiero islámico no cambiaría significativamente. (Khan \& Mirakhor 1989).

En consecuencia, Khan y Mirakhor continuaron sus trabajos en esta dirección y en 1994 admitieron que la política monetaria del sistema financiero islámico puede coexistir con el sistema financiero convencional. Po ello, las autoridades monetarias pueden utilizar todos los instrumentos convencionales, excepto la tasa de interés, y cualquier otro instrumento que implique la tasa de interés. Pueden utilizar las operaciones de mercado abierto, en las que se negocian participaciones de capital propio en lugar de bonos, así como el crédito, las reservas obligatorias y los coeficientes de participación en los beneficios, aunque los estudiosos musulmanes siguen teniendo opiniones encontradas sobre estas herramientas.

Este es el caso del consejo pakistaní que, durante su misión sobre la eliminación de las prácticas usureras en el sistema bancario, consideró que la función del banco central sería la misma, por lo que la mayoría de los instrumentos no se verían afectados, a excepción del tipo de descuento que no sirve para la actividad bancaria islámica. Se sustituiría por ratios de participación en los beneficios. (Ziauddin \& al, 1983).

Este punto de vista es totalmente rechazado por Chapra (1997), quien considera que para aplicar una política monetaria islámica, no sólo hay que abandonar la tasa de interés, sino también las operaciones de mercado abierto.

En su análisis, Choudhry y Mirakhor (1997) proporcionaron una explicación más detallada de los instrumentos monetarios directos y indirectos, al tiempo que distinguen entre los instrumentos que pueden ser aplicados por la política monetaria islámica y los que deberían excluirse.

De hecho, reconocen que la mayoría de los instrumentos directos de la política monetaria convencional pueden ser utilizados por la política monetaria islámica, ya que no todos se basan en las tasas de interés y, por lo tanto, se ajustan a la Shariah. A diferencia de los instrumentos indirectos, que 
tienen un interés fijo y, por lo tanto, están excluidos del concepto monetario islámico.

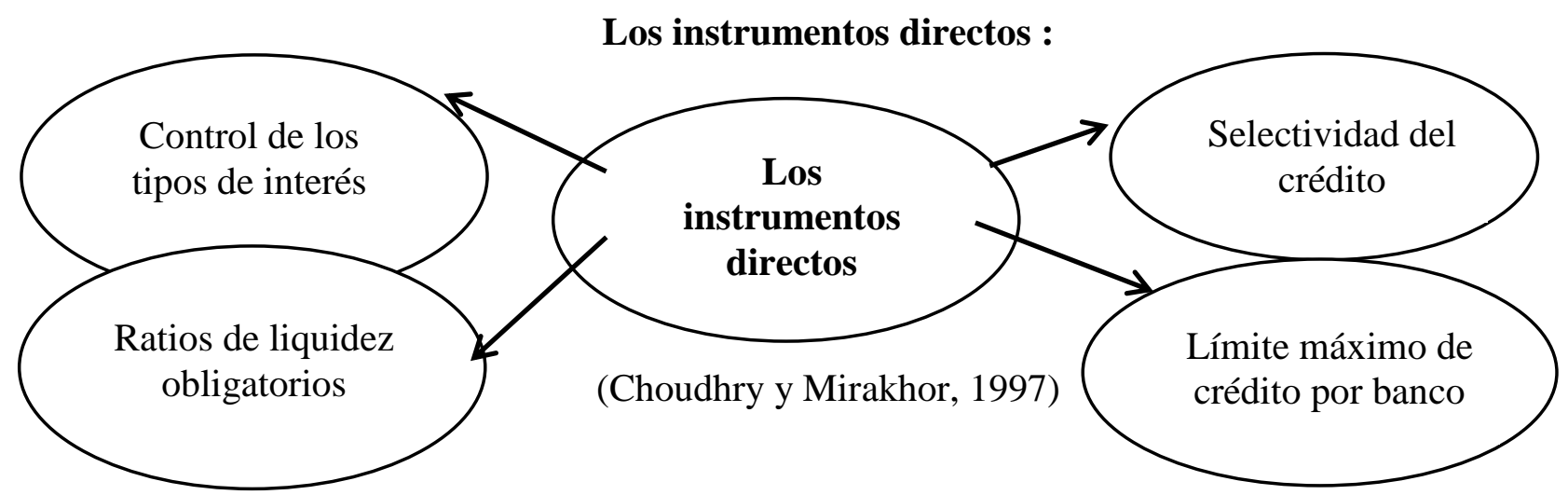

Dado que la tasa de interés no debería ser parte de los instrumentos de la política monetaria islámica, el banco central iraní la reemplazó con la tasa de beneficio bancaria controlada. De hecho, los bancos islámicos operan con contratos de rendimiento fijo y contratos participativos como el Musharakah, por lo que el banco invierte los depósitos de los depositantes en proyectos y les asigna su parte en forma de beneficios. Sin embargo, estos proyectos suelen ser a largo plazo y, por lo tanto, el banco proporciona a los depositantes beneficios anuales o mensuales y, después de dos años o más, cuando el banco puede calcular sus beneficios exactos, les da la cantidad exacta. De esta forma, el banco central controla indirectamente los depósitos bancarios. Cuando opta por una política monetaria expansiva, reduce la tasa de beneficio del sistema bancario y disuade a los depositantes de colocar su dinero en depósitos bancarios. Este control asegura que el banco central tenga un seguimiento cuidadoso de los depósitos bancarios. (Kiaee, 2007)

En efecto, los instrumentos directos son inherentes a los activos bancarios. Controlan eficazmente la asignación y distribución del crédito bancario de acuerdo con las directrices del banco central.

Esta panoplia de instrumentos se recomienda específicamente para los sistemas financieros que acaban de iniciar la actividad financiera islámica junto con el sistema convencional. Se caracterizan por su fiabilidad en el control de los agregados crediticios. De hecho, han demostrado un excelente rendimiento en caso de crisis financiera, especialmente en países con sistemas financieros menos desarrollados. Su principal cualidad es que son fáciles de aplicar y evaluar.

Sin embargo, tienen ciertas limitaciones relacionadas principalmente con sus contribuciones a la contención del progreso que el sector privado puede hacer en el sector bancario, especialmente cuando el sistema bancario está dominado por los bancos estatales. En los países desarrollados, la experiencia de los instrumentos directos demuestra que su eficacia se 
desvanece con el tiempo debido a que se eluden de muchas maneras. De hecho, los bancos pueden ser responsables del debilitamiento de los instrumentos de control directo al fomentar nuevas técnicas de financiación que no están sujetas a las herramientas de control aplicadas y desvían fondos hacia actividades artificialmente rentables creadas por los propios controles. Por ello, el rendimiento de los instrumentos directos puede ser a menudo engañoso.

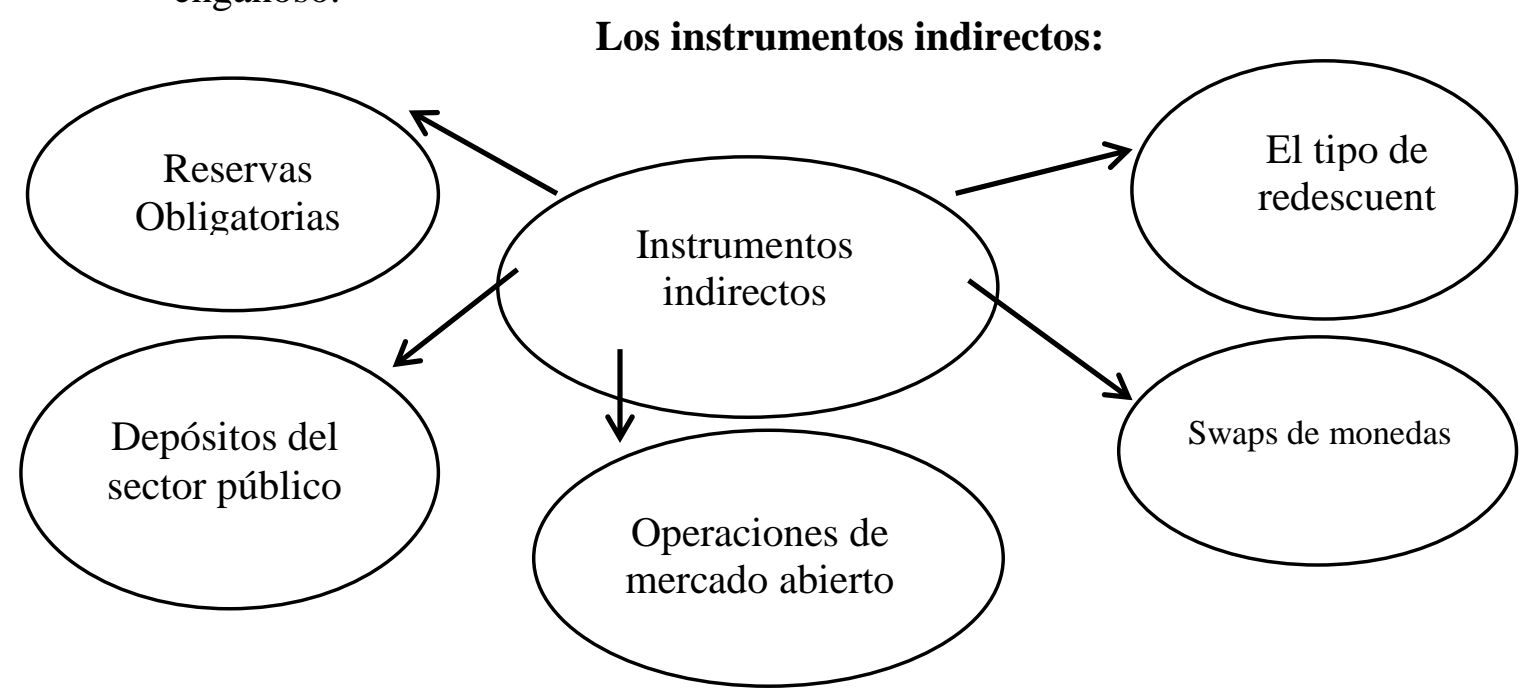

(Choudhry y Mirakhor, 1997)

La razón principal de la existencia de los instrumentos indirectos es llenar las lagunas de los instrumentos directos, que con el tiempo pierden sus eficacias. Así, los instrumentos indirectos tienen la capacidad de controlar las fluctuaciones de liquidez a corto plazo en consonancia con los objetivos de la política monetaria. Las reservas obligatorias y los depósitos tienen un impacto directo en los saldos de reserva de los bancos con el banco central. Por otro lado, los swaps de divisas pueden iniciar un cambio en la composición de los activos denominados en moneda extranjera y en moneda nacional. También pueden influir en los saldos de reserva de los bancos con el banco central a través del mecanismo de de compra y venta de divisas.

En cuanto a las operaciones de mercado abierto, proporcionan un control monetario más eficaz que el que ofrecen los instrumentos indirectos, porque se caracterizan por una notable flexibilidad y son capaces de modificar las condiciones monetarias incluso cuando determinados agregados monetarios pierden importancia económica. A pesar de sus contribuciónes al desarrollo de los mercados financieros, se consideran instrumentos bastante complejos y con una gran ambigüedad sobre sus impactos en los agregados monetarios. 
Además, si las exigencias de reservas no se distribuyen de manera equitativa entre los bancos, no pueden absorber el exceso de liquidez. Por lo tanto, se produce una expansión excesiva del crédito si los depósitos a la vista están sujetos a requisitos de reserva del $100 \%$, ya que los requisitos de reserva para los depósitos de inversión serían escasos o nulos.

Las limitaciones de los depósitos del sector público están relacionadas principalmente con la falta de transparencia, que tiene un impacto negativo en el desarrollo de los mercados financieros. Por lo tanto, los instrumentos indirectos presentan muchas limitaciones en comparación con los sistemas financieros islámicos. (Choudhry y Mirakhor, 1997)

Por ello, varios investigadores han propuesto instrumentos adecuados a los principios de las finanzas islámicas como sustitutos. En particular, Choudhry y Mirakhor (1997) proponen una alternativa real a estos instrumentos, en forma de títulos públicos basados en la participación en los beneficios con tasas de rendimiento basadas en el superávit presupuestario. Estos tipos se ajustan a la sharia. Actúan como técnicas indirectas de control monetario y se asimilan a las operaciones de mercado abierto.

Más recientemente, Bidabad (2019) reconoce la inutilidad de los instrumentos monetarios del sistema bancario convencional en la política monetaria del sistema islámico, ya que el instrumento más influyente de la política monetaria, las operaciones de mercado abierto, no están permitidas. Por ello, propone un sustituto de las operaciones de mercado abierto sin intereses, que son los "Bonos Swap de Rastin", que respetan el derecho del prestamista a tomar prestada una cantidad igual a su préstamo del prestatario. En caso contrario, adopta la forma de una deuda igual a un préstamo futuro o de un préstamo igual a una deuda futura. De acuerdo con la Shariah, no se fija ningún tipo de interés a nivel de este bono, sin embargo su precio se determina en función del precio de mercado sin que el rendimiento del bono esté fijado o predeterminado. Este instrumento se está tramitando en Irán, pero aún no se ha puesto en marcha, por lo que sus implicaciones prácticas aún no se evalúan (Bidabad 2019)

Rifki Ismal (2011) parte del principio de que el sistema financiero islámico necesita instrumentos específicos a su naturaleza, así, su estudio es el primero en proponer instrumentos específicos para el modelo islámico de acuerdo con la Sharia, estos instrumentos responden a necesidades específicas a saber :

- El certificado Wakalah wa Ijarah :con el objetivo principal de resolver el problema de liquidez mediante el uso de fondos no utilizados en lugar de inyectar nueva liquidez. La contracción monetaria a nivel de esta herramienta, corresponde al principal del certificado, y la 
expansión del dinero representa la compra del activo, el pago de los rendimientos y el reembolso del principal.

- El certificado de Wakalah wa Ijarah Muntahia Bitamlik :Es similar al anterior. Con el objetivo de lograr un mayor impacto expansivo, el pago regular a los depositantes incluye el pago de los rendimientos y el reembolso del principal. Si el gobierno es el arrendatario de dicho activo, la transferencia de propiedad que genera es beneficiosa para la transacción monetaria.

- El certificado Musharakah Mutanaqisah wa Ijarah :Tiene como objetivo la absorción de liquidez porque los titulares de los certificados no reciben sus ingresos regularmente del banco central. El gobierno tendrá la ventaja de convertirse en el inquilino del proyecto. Así, la contracción monetaria en este instrumento está representada por la cuota de los inversores en un proyecto y la expansión monetaria está representada por los gastos necesarios para la ejecución de un proyecto.

- El certificado islámico de titulización wa Ijarah :Es similar al anterior. Hay un impacto expansivo real cuando el banco central libera sus ingresos regulares de Ijarah a los depositantes para recuperar los activos. Para controlar mejor el impacto del exceso de liquidez a corto plazo, el banco central debería gestionar con precisión el tiempo entre la contracción y la expansión de este instrumento. (Ismal, 2011)

\subsection{Los canales de transmisión de la política monetaria: El canal del tipo de interés:}

Es el actor fundamental en la transmisión de la política monetaria en la corriente keynesiana. Bajo el supuesto de la rigidez de los precios, este canal se caracteriza por la importancia que otorga a la tasa de interés real en lugar de la nominal, como la que influye en la actividad económica. Cuando el banco central ejerce cambios en el tipo de interés nominal a corto plazo, el tipo de interés real a corto y largo plazo cambia sistemáticamente. Este proceso se explica por la rigidez de los precios, de modo que una política monetaria encaminada a reducir la tasa de interés nominal a corto plazo también baja la tasa de interés real de corto plazo. (Mishkin, 1996)

Sin embargo, los bancos centrales no son los únicos que controlan la creación de dinero o los tipos de interés. De hecho, los bancos convencionales también son capaces de crear dinero mediante la creación de deuda.

En la actualidad, la mayoría de los bancos islámicos se encuentran en países con sistemas financieros duales, que operan uno junto al otro a los bancos convencionales en mercados monetarios muy sofisticados. En consecuencia, los bancos islámicos operan en sistemas financieros en los que los tipos de interés son dominantes, lo que repercute en los costes de 
financiación y el rendimiento de las cuentas de inversión de los bancos islámicos. Por esta razón, algunos bancos islámicos utilizan los tipos de interés para fijar las condiciones de los contratos Murabahah e Ijara.

Sin embargo, a pesar de este enfoque, parece que los bancos islámicos pueden verse influidos por las variaciones de los tipos de interés, por lo que la transmisión de la política monetaria a través de los tipos de interés será menos eficaz, especialmente en presencia de grandes bancos islámicos. (Khatat, 2016)

\section{El canal del tipo de cambio :}

Con el crecimiento de la internacionalización económica, el tipo de cambio se ha convertido en uno de los principales actores en la transmisión de la política monetaria. Además, este canal interactúa con la transmisión de los tipos de interés. Así, la disminución de los tipos de interés conduce a una depreciación de los depósitos nacionales frente a los depósitos en moneda extranjera, lo que resulta en la depreciación de la moneda nacional. Por lo tanto, el tipo de cambio desempeña un papel crucial en la forma en que la política monetaria afecta a la economía nacional. (Mishkin, 1996)

De hecho, los bancos islámicos hoy operan en países que aplican un tipo de cambio fijo o un régimen de objetivos monetarios, como los países del Consejo de Cooperación del Golfo, Jordania y Brunei, mientras que Bangladesh y Yemen permiten una flexibilidad limitada del tipo de cambio. Además, los países con bancos islámicos y tipos de cambio flexibles se caracterizan por una inflación más estable y un crecimiento menos estable que los países con tipos de cambio más flexibles. (Khatat, 2016)

\section{$>$ El canal de crédito:}

La tasa de interés y el tipo de cambio no son los únicos canales a través de los cuales la política monetaria impacta la actividad económica, el canal del crédito es también una herramienta determinante en la transmisión de la política monetaria. La teoría distingue entre dos tipos de canales de crédito, el primero es el canal de crédito bancario que se basa en el multiplicador de crédito y que pierde su eficacia con la liberalización financiera, cuando los bancos acceden fácilmente a los recursos financieros externos. (Khatat, 2016)

El segundo se refiere al canal del balance, que se refiere a la situación financiera de los posibles prestatarios. (Creel y Levasseur, 2006).

Para los países con sistemas financieros duales, los saldos de reservas pueden desempeñar un papel fundamental en la conducción de la política monetaria. En consecuencia, el canal de crédito o financiación de los bancos islámicos depende del grado en que el banco central sea capaz de proporcionar liquidez a los bancos islámicos y de la capacidad de estos últimos para reorientar su oferta de crédito / financiación en respuesta a las variaciones de sus saldos de reserva en el banco central. Sin embargo, este canal se vuelve ineficaz cuando los bancos islámicos o convencionales tienen un exceso de 
liquidez o cuando la oferta y demanda de crédito están limitadas. (Khatat, 2016)

\section{Instrumentos de política monetaria: un estudio exploratorio Metodología :}

Para analizar mejor los instrumentos de la política monetaria, realizamos un estudio exploratorio a través del análisis de los bancos centrales de los países del CCG y de Irán, con el fin de extraer las herramientas con las que los bancos centrales dirigen la política monetaria en los dos casos de forma diferenciada. El siguiente cuadro (Cuadro 1) presenta los diferentes países incluidos en el estudio, así como la naturaleza de sus sistemas financieros:

Cuadro 1 : La lista de países del estudio exploratorio y la naturaleza de sus sistemas financieros

\begin{tabular}{|c|c|}
\hline País & $\begin{array}{c}\text { Naturaleza del sistema } \\
\text { financiero } \\
\text { Islamizado }\end{array}$ \\
\hline Irán & Dual \\
\hline Bahrain & Dual \\
\hline Arabia Saudí & Dual \\
\hline Kuwait & Dual \\
\hline Qatar & Dual \\
\hline Omán & Dual \\
\hline Emiratos Árabes Unidos & \\
\hline
\end{tabular}

\section{1. $\quad$ El caso de Irán : Principales Resultados}

En 1984 se aprobó y entró en vigor la ley que prohíbe las prácticas usurarias en Irán. A diferencia de la experiencia pakistaní, que se puso en práctica de forma transitoria, Irán optó por la conversión de todo el sistema financiero.

La supresión del tipo de interés está en el centro de esta ley, por lo que la estructura de los balances bancarios ha sufrido cambios. En consecuencia, las dos cuentas que se han permitido son las cuentas de ahorro sin intereses y los depósitos de inversión a plazo con tipos de beneficio predefinidos. (Coville, 1994)

En consecuencia, los instrumentos directos e indirectos de la política monetaria se han adaptado para cumplir con la sharia. Son los siguientes: 
Cuadro 2: Instrumentos directos del Banco Central de Irán

\begin{tabular}{|c|c|}
\hline Instrumentos & Operación \\
\hline Tipo de beneficio bancario & $\begin{array}{l}\text { Tras la aplicación de la ley de bancos } \\
\text { sin interés y la introducción de los contratos } \\
\text { de rentabilidad fija y los contratos } \\
\text { participativos, los dispositivos que permiten } \\
\text { determinar la tasa de ganancia y la tasa de } \\
\text { rentabilidad de los establecimientos } \\
\text { bancarios son determinados por el Consejo } \\
\text { de Dinero y crédito. Además, el banco } \\
\text { central iraní puede intervenir en la } \\
\text { determinación de estas tasas tanto para } \\
\text { proyectos de inversión o de asociación como } \\
\text { para otras facilidades otorgadas por los } \\
\text { bancos. }\end{array}$ \\
\hline Techo de crédito & $\begin{array}{l}\text { De acuerdo con el artículo } 14 \text { de la ley } \\
\text { monetaria y bancaria iraní, el Banco Central } \\
\text { de Irán interviene para identificar a los } \\
\text { bancos el perímetro de uso de los fondos y } \\
\text { determinar el límite de préstamos y créditos } \\
\text { para cada sector de actividad. }\end{array}$ \\
\hline
\end{tabular}

Cuadro 3: Instrumentos indirectos del Banco Central de Irán

\begin{tabular}{|c|c|}
\hline Instrumentos & Operación \\
\hline $\begin{array}{l}\text { Coeficiente de reservas } \\
\text { obligatorias }\end{array}$ & $\begin{array}{l}\text { Los bancos están obligados a depositar una parte de sus } \\
\text { pasivos en forma de depósito en el Banco Central de Irán. } \\
\text { Según el artículo } 14 \text { de la Ley Monetaria y Bancaria } \\
\text { iraní, el banco central está autorizado a determinar este ratio } \\
\text { entre el } 10 \text { y el } 30 \% \text { en función de la composición de los } \\
\text { pasivos de los bancos y del sector de actividad. }\end{array}$ \\
\hline $\begin{array}{c}\text { Documentos de } \\
\text { participación del banco central }\end{array}$ & $\begin{array}{l}\text { Tras la implementación de la ley bancaria sin intereses, } \\
\text { se hizo necesario adaptar los instrumentos monetarios a los } \\
\text { preceptos de la sharia. Las operaciones de mercado abierto } \\
\text { han sido reemplazadas por el uso de títulos de participación } \\
\text { y sociedades de inversión. } \\
\text { Con este instrumento, el banco central iraní puede } \\
\text { ajustar el dinero en sentido amplio (M2) a través de la base } \\
\text { monetaria y así puede controlar la tasa de inflación. }\end{array}$ \\
\hline $\begin{array}{c}\text { Abrir una cuenta de } \\
\text { depósito especial en el banco } \\
\text { central }\end{array}$ & $\begin{array}{l}\text { Debido a la supresión de los intereses en su actividad } \\
\text { bancaria, Irán utiliza la apertura de una cuenta especial de } \\
\text { depósito en el banco central como uno de los instrumentos } \\
\text { indirectos de su política monetaria. Este instrumento ayuda } \\
\text { a controlar la liquidez absorbiendo el exceso de recursos de } \\
\text { los bancos. Por lo tanto, el banco central paga beneficios a } \\
\text { estos depósitos según normas específicas. }\end{array}$ \\
\hline
\end{tabular}

(El Banco Central de Irán) 
Como hemos detallado en la revisión de la literatura, aparte del tipo de interés, los instrumentos directos convencionales pueden utilizarse en el sistema financiero islámico, ya que no todos se basan en el tipo de interés. A diferencia de los instrumentos monetarios indirectos, que en su mayoría se basan en los tipos de interés, los sistemas financieros islámicos deberían sustituirlos por herramientas conformes a la sharia. Este es el caso de Irán, como se indica en las tablas anteriores (Cuadro 2 y 3).

\subsection{Caso de un sistema financiero dual: coexistencia de la banca islámica y convencional}

En los sistemas financieros duales, la relación entre el banco islámico y el banco central es muy importante. De hecho, este último es el proveedor de liquidez para los bancos islámicos en caso de necesidad. Además, la confianza del público se basa principalmente en esta relación, porque mientras el banco central mantenga este tipo de intervención con el banco islámico, tranquiliza a los clientes principalmente en tiempos de crisis. (Mira, 2011).

El análisis de los bancos centrales de los países del CCG revela los siguientes resultados. De hecho, (el cuadro 4) ofrece respuestas a las siguientes preguntas:

- Cuando los bancos islámicos y los bancos convencionales coexisten en un sistema financiero, ¿existen instrumentos monetarios adecuados según los preceptos de la sharia? En caso afirmativo, ¿cuáles?

- ¿Y cuáles son los otros instrumentos con los que los bancos centrales acompañan la política monetaria? 
Cuadro 4 : Los resultados del estudio exploratorio por países

\begin{tabular}{|c|c|c|c|}
\hline País & $\begin{array}{l}\quad \text { ¿Existen } \\
\text { instrumentos } \\
\text { monetarios } \\
\text { islámicos? }\end{array}$ & ¿Cuáles? & $\begin{array}{l}\text { Instrumentos convencionales de } \\
\text { política monetaria }\end{array}$ \\
\hline Bahrain & Sí & $\begin{array}{ll}\text { - } & \text { Wakalah conforme } \\
& \text { a la sharia } \\
\text { - } & \text { Sukuk-al-ijara } \\
\text { - } & \text { Sukuk-al-Salam }\end{array}$ & $\begin{array}{ll}- & \text { Operaciones de mercado abierto } \\
\text { - } & \text { Las exigencias de reservas } \\
\text { - } & \text { Tipo de cambio fijo }\end{array}$ \\
\hline Arabia Saudí & Sí & - $\quad$ Sukuks & $\begin{array}{ll}- & \text { La proporción dereserva de } \\
\text { dinero en efectivo (CRR) } \\
\text { - } & \text { Repos } \\
\text { - } & \text { Swaps de divisas } \\
\text { - } & \text { Colocación de fondos públicos }\end{array}$ \\
\hline Kuwait & Sí & $\begin{array}{ll}\text { - } & \text { Operaciones de } \\
\text { Tawarruk }\end{array}$ & $\begin{array}{ll}\text { - } & \text { Tipo de cambio fijo } \\
\text { - } & \text { Subasta de depósitos } \\
\text { - } & \text { Límites máximos de los } \\
& \text { préstamos bancarios }\end{array}$ \\
\hline Qatar & Sí & - $\quad$ Sukuks & $\begin{array}{ll}\text { - } & \text { Operaciones de mercado abierto } \\
\text { - } & \text { Coeficiente de reservas exigido } \\
\text { - } & \text { La ventana de descuento } \\
\text { - } & \text { Mercado monetario de Qatar } \\
\text { - } & \text { Operaciones de recompra de } \\
& \text { débito público-Repo }\end{array}$ \\
\hline $\begin{array}{c}\text { Emiratos } \\
\text { Árabes Unidos }\end{array}$ & Sí & $\begin{array}{l}\text { - Línea Murabaha } \\
\text { colateralizada } \\
\text { - Certificados de } \\
\text { depósito islámicos }\end{array}$ & $\begin{array}{l}\text { - As exigencias de reservas minimas } \\
\text { - Los certificados de depósito } \\
\text { - Repo y amortización anticipada del } \\
\text { certificado de depósito } \\
\text { - Swaps de divisas } \\
\text { - Mecanismo de crédito }\end{array}$ \\
\hline Omán & No & - & $\begin{array}{l}\text { - } \quad \text { La vinculación de la moneda } \\
\text { - Requisitos de reserva } \\
\text { - Repo/ Operaciones de repo } \\
\text { inversa } \\
\text {-Descuento/ redescuento de efectos } \\
\text { comerciales } \\
\text {-Certificados de depósito } \\
\text { - Descuento de Letras del Tesoro } \\
\text { - Swaps de divisas } \\
\text { - Préstamo directo }\end{array}$ \\
\hline
\end{tabular}

De hecho, es evidente que los países del CCG han realizado algunos esfuerzos notables para emitir instrumentos financieros conformes con la sharia para satisfacer las necesidades de liquidez e inversión de los bancos y para hacer frente a la segmentación entre los mercados bancarios convencionales e islámicos. 
De esta tabla, podemos ver que los países en los que los bancos convencionales operan junto a los bancos islámicos han puesto en marcha instrumentos monetarios para apoyar la actividad de los bancos islámicos, con la excepción de Omán, que todavía no ha puesto en marcha ningún instrumento monetario islámico.

Para los países que han optado por operar a través de un sistema financiero dual, la elección de los instrumentos monetarios islámicos sigue siendo limitada, principalmente los Sukuks y las operaciones Tawarruq. Sin embargo, los EAU parecen tener una seria ambición hacia el desarrollo de instrumentos monetarios islámicos a través de una ingeniería financiera innovadora.

\section{Conclusión}

Para cumplir con los objetivos de estabilidad monetaria y financiera, se supone que el sistema financiero islámico, al igual que su homólogo convencional, está sujeto a las mismas condiciones en materia de política monetaria. Para ello, el banco central utiliza varios instrumentos monetarios para regular la cantidad de dinero en circulación.

Sin embargo, cuando los dos sistemas financieros coexisten, la práctica de la política monetaria constituye un reto que invita a la reflexión. De hecho, la prohibición de ciertas prácticas, como el uso de tipos de interés, por parte de las finanzas islámicas crea una necesidad real de diseñar instrumentos adecuados a los preceptos de la Sharia.

Así pues, la coexistencia de los bancos islámicos con los bancos convencionales requiere el desarrollo de instrumentos monetarios conformes a la sharia para mejorar la eficacia de las operaciones monetarias. Un fuerte compromiso de los bancos convencionales en este sentido igualaría las condiciones.

Los sistemas financieros duales suelen caracterizarse por el desarrollo del sector bancario convencional más que el islámico. Esto dificulta el funcionamiento de la política monetaria cuando los dos sistemas se encuentran en diferentes niveles de desarrollo.

\section{References:}

1. Ahmad, A. U. F., \& Hassan, M. K. (2006). The Time Value of Money Concept in Islamic Finance. American Journal of Islam and Society, 23(1), 66-89.

2. Ayub, M. (2007). Understanding Islamic finance. Hoboken, NJ: John Wiley \& Sons.

3. Bidabad, B. (2019). Islamic Monetary Policy and Rastin Swap Bonds. International Journal of Islamic Banking and Finance Research, 3(2). 
4. Chapra, M.U. (1997). Vers Un Système Monétaire Juste. Djeddah : Institut Islamique de Recherche et de Formation- Banque Islamique de Développement.

5. Choudhry, N.N., \& Mirakhor, A. (1997). Indirect Instruments Of Monetary Control In An Islamic Financial System. Islamic Economic Studies, 4, 28-65.

6. Coville, T. (1994). Le système financier islamique en Iran : de la rhétorique à la pratique. Cahiers d'études sur la Méditerranée orientale et le monde turco-iranien, 17, 287-292.

7. Creel, J. \& Levasseur, S. (2006). Canaux de transmission de la politique monétaire dans l'ue: Le cas de trois nouveaux entrants. Revue économique, 4(4), 881-898. https://doi.org/10.3917/reco.574.0881

8. Ismal, R. (2011). Central bank Islamic monetary instruments: a theoretical approach. Studies in Economics and Finance, 28, 51-67.

9. Khan, M. S., \& Mirakhor, A. (1989). The Financial System and Monetary Policy in an Islamic Economy. Journal of King Abdulaziz University: Islamic Economics, King Abdulaziz University, Islamic Economics Institute, 1(1), 39-57.

10. Khan, M. S., \& Mirakhor, A.(1994). Monetary Management in an Islamic Economy . Journal of King Abdulaziz University: Islamic Economics, 6(1).

11. Khatat, E. M., (2016). Monetary Policy in the Presence of Islamic Banking. IMF Working Papers, Article WP/16/72.

12. Kiaee, H.(2007). Monetary Policy In Islamic Economic Framework: Case of Islamic Republic of Iran.

13. Mira, H.(2011). Study revealing that the Islamic financing extends the participation in ownership of projects and integration. Middle East newspaper, 11759.

14. Mishkin, F. (1996). Les canaux de transmission monétaire : Leçons pour la politique monétaire. Bulletin de la Banque de France, 27.

15. Ziauddin, A., Iqbal, M.,\& Khan, F.(1983).[Review of the book Money and Banking in Islam, by M. Hassanein]. Journal of Research in Islamic Economics, 3(1), 93-99

16. Zangeneh, H., \& Salam, A.(1993). Central Banking in an Interest-Free Banking System. Journal of King Abdulaziz University: Islamic Economics,5,25-36. 